\title{
Blood Ammonia level : Is it a Clue for the Presence of Oesophageal Varices in Cirrhotic Patients ?
}

\author{
Lashin, A.H ${ }^{1}$; El feky, H.M ${ }^{1}$; Elbehisy, M.M² Nada, M.E $^{3}$ \\ ${ }^{1}$ Hepatology, Gastroenterology and Infectious Diseases Department, Faculty of Medicine, \\ Benha University, Egypt. \\ ${ }^{2}$ Clinical Pathology Department,Faculty of Medicine,Benha University, Egypt. \\ ${ }^{3}$ Hepatology, Gastroenterology and Infectious Diseases Department, \\ Shebein El-Kom Teaching Hospital-Shebein El-Kom, Egypt.
}

Corresponding Author

Hala Mohammad El

Feky

Mobile:

$+201006432009$

E mail:

halaelfeky-

nad@hotmail.com

Key words:

Oesophageal varices

(OV); Blood ammonia level $(B A L)$; Spleen

longitudinal

diameter(SLD); Portal

vein diameter $(P V D)$;

Splenic vein

diameter(SVD)
Background and study aim: Endoscopic screening of all patients with liver cirrhosis add major burden to endoscopic units. Non-invasive detection of Oesophageal varices (OV) help to reduce the necessity of endoscopic screening. The aim of this work is to assess the diagnostic utility of blood ammonia level (BAL) as noninvasive predictor for presence of $\mathrm{OV}$ and evaluate its correlation with variceal size in cirrhotic patients.

Patients and Methods: This prospective cross sectional study was conducted upon 100 cirrhotic patients who attended Hepatology, Gastroenterology and Infectious diseases department, Benha University Hospital. Fasting blood ammonia was measured and upper gastrointestinal endoscopy was done for all patients. Patients were divided according to presence of OV into two groups: Group $\mathrm{I}$ : included 30 patients with liver cirrhosis without OV. Group II: included 70 patients with liver cirrhosis with $\mathrm{OV}$ who were subdivided into four subgroups: Group IIa:included 11 patients with grade I O.V. Group IIb: included 21 patients with grade II O.V. Group IIc: included 15 patients with grade III O.V. Group IId: included 23 patients with grade IV O.V.

Results: The study showed that there was a highly significant increase in the mean values of BAL in cirrhotic patients with varices in comparison to those without varices. Also the study showed highly significant increase in the mean values of BAL in patients with large OV (grade III,IV) in comparison to patients with small and medium sized varices (grade I,II). By multivariate analysis, the presence of O.V. was independently associated with increased blood ammonia levels.

Conclusion: Blood ammonia level could be a non invasive predictor for the presence of $\mathrm{OV}$ and could be clinically useful, as it correlated with the size of OV.

\section{INTRODUCTION}

Liver cirrhosis is a leading cause of death worldwide. It is the end result of a long-lasting process, usually clinically silent and unnoticed by the patient and the physician for years [1]. The clinical course of patients with advanced cirrhosis is often complicated by a number of important sequelae that can occur regardless of the underlying cause of the liver disease. These include portal hypertension and its consequences of gastroesophageal variceal hemorrhage [2]. Portal hypertension is a progressive, inevitable sequelae of liver cirrhosis that leads to formation of portosystemic collateral veins, among them, oesophageal varices (OV) have the greatest clinical influence because their rupture results in variceal bleeding that can be fatal. OV can be diagnosed by Upper gastrointestinal (GI) endoscopy which is the gold standard and it is recommended by guidelines to screen all cirrhotic patients for $\mathrm{OV}$ at the time of diagnosis. Lack of detection of $\mathrm{OV}$ at the first endoscopic evaluation mandates repeat endoscopy annually in decompensated cirrhotic patients and every 2-3 years in patients with compensated cirrhosis [3]. However, the majority of cases undergoing screening endoscopy either do not have varices or have 
varices but do not require prophylactic therapy [4]. Patients without varices develop them at a rate of $8 \%$ per year and the progression from small to large varices occurs in 10 to $20 \%$ of cases yearly [5] Therefore, the identification of non endoscopic, non invasive methods that can accurately predict OV in cirrhotic patients, particularly those of large size, can help to identify patients at greatest risk and hence reduce the exigency of endoscopic screening [6]. In cirrhosis, the major portion of ammonia carried by portal blood is shunted by portosystemic collaterals into systemic circulation. This raised blood ammonia level (BAL), on the other hand, could be a good mirror of portosystemic collaterals as well as portal hypertension [7]. The aim of this study was to evaluate the utility of blood amonia as a non invasive predictor of $\mathrm{OV}$ in cirrhotic patients regarding their presence and size and compared it with platelet count/ splenic diameter ratio that is one of the most important non invasive predictors of O.V.

\section{PATIENTS AND METHODS}

\section{Study design}

This cross-sectional study was carried out prospectively at the department of Hepatology, Gastroenterology and Infectious diseases, Benha University Hospital. The study was approved by ethical committee of Benha Faculty of Medicine and its University Hospitals. Before enrolling in the study, informed consent was obtained from each participant.

\section{Patients}

Hundred adult cirrhotic patients were included in this study. All patients met the diagnostic criteria of liver cirrhosis by clinical, biochemical and ultrasonographic findings. Patients were divided according to the results of the upper gastrointestinal endoscopy into the following groups:

- Group I: Included 30 patients with liver cirrhosis with no endoscopic evidence of oesophageal varices.

- Group II: Included 70 patients with liver cirrhosis with endoscopic evidence of oesophageal varices, who were subdivided into four subgroups according to the Modified Grading System [8] :

$\checkmark$ Group IIa: Included 11 patients with grade I O.V.

$\checkmark$ Group IIb: Included 21 patients with grade II O.V. $\checkmark$ Group IIc: Included 15 patients with grade III O.V.

$\checkmark$ Group IId: Included 23 patients with grade IV O.V.

\section{Exclusion criteria :}

Patients who received endoscopic variceal ligation (EVL) or sclerotherapy, surgery for oesophageal varices, patients with history of previous or current use of beta blockers, presence of hepatic encephalopathy, active or recent GI bleeding within 4 weeks, portal vein thrombosis on ultrasonography, hepatocellular carcinoma, serum creatinine of $>1.3 \mathrm{mg} / \mathrm{dl}$ and patients in whom endoscopy is contraindicated were excluded from the study.

Patients were then evaluated by thorough history taking, complete clinical examination (to confirm signs of chronic liver disease or liver cell failure and signs of complications), Complete blood count (CBC), Liver profile tests including; Serum Albumin (gm/dl), Prothrombine time and activity, Alanine aminotransferase (ALT) (U/L), Aspartate aminotransferase (AST) (U/L), Serum bilirubin (total and direct) $(\mathrm{mg} / \mathrm{dl})$, Kidney function tests including; Blood urea $(\mathrm{mg} / \mathrm{dl})$ and Serum Creatinine $(\mathrm{mg} / \mathrm{dl})$, Blood levels of ammonia (NH4) $(\mu \mathrm{mol} / \mathrm{l})$ : measured by kinetic enzymatic method with glutamate dehydrogenase by using ammonia-liquizyme single reagent [9] provided by Bioassay system, Germany. Abdominal ultrasonographic examination was done with stress on portal vein diameter, spleen longitudinal diameter and exclusion of hepatocellular carcinoma and portal vein thrombosis. Upper GIT endoscopy was done by olympus video endoscopy at Hepatology, Gastroenterology and Infectious diseases department, Benha University Hospital. During endoscopy, presence of esophageal varices and its grades, gastric varices, portal hypertensive gastropathy were noted. Modified Child Pugh Score was calculated for all patients.

\section{Statistical analysis :}

The data collected were tabulated and analyzed by SPSS (statistical package for social science) version 22.0 on IBM compatible computer.

Two types of statistics were done:

I- Descriptive 1- Mean value (X) and Standard Deviation [SD]: for quantitative data. 2Frequency and percenatage for qualitative data.

II- Analytic by t-student test and Chi-square test. $\mathbf{P}$ value $>0.05$ was considered statistically non significant $\mathbf{P}$ value $\leq 0.05$ was considered 
statistically significant. $\mathbf{P}$ value $\leq 0.001$ was considered statistically highly significant. Variables found to be associated with the dependent variable at univariate logistic regression at a probability threshold of less than 0.10 were entered into multivariate logistic regression models to avoid the effect of co linearity.

\section{RESULTS}

This prospective study was conducted upon 100 adult cirrhotic patients who were classified into two groups; group 1 included 30 cirrhotic patients without esophageal varices and group 2 included 70 cirrhotic patients with esophageal varices. The mean age of group 2 cases was $48.93 \pm 9.84$ which was significantly higher than that of group $1($ Mean $\pm S D=42.67 \pm 8.70)$. Male predominance was noted in group $2 ; 52$ cases (74.29\%) vs. 14 cases (46.67\%) in group 1 with statistical significant difference between both groups. Regarding Child classification, most patients in group I were Child A $(66.7 \%)$ and most patients in group II were Child $\mathrm{B}$ and $\mathrm{C}$ (74.29\%). BAL was significantly higher and platelet count/splenic diameter ratio was significantly lower in group 2 than group 1 (p value $=0.00$ for each). On the same hand, BAL was significantly higher and platelet count/ splenic diameter ratio was significantly lower in large sized varices (grade III and IV) than small sized varices (grade I and II). BAL was the only predictor of presence of $\mathrm{OV}$ by multivariate logistic regression analysis. Spearman correlation revealed that there was highly significant positive correlation between BAL and size of varices $(\mathrm{P}$ value $<0.001)$. BAL at cutoff value $=$ $48 \mathrm{umol} / \mathrm{L}$ had $75.7 \%$ sensitivity, $100 \%$ specificity, 100\% PPV, $63.8 \%$ NPV and $93.2 \%$ accuracy in detection of OV and at cutoff level $67 \mathrm{umol} / \mathrm{L}$, it had $46.2 \%$ sensitivity, $97.7 \%$ specificity, $75 \%$ PPV and 92.4\% NPV for detection of large OV (grade III and IV). Platelet count $/$ splenic diameter ratio at cutoff level $=$ 1073 had sensitivity $94.3 \%$, specificity $56.7 \%$, PPV $83.5 \%$ and NPV $81 \%$ for detection of OV and at cutoff level $=570$ it had $76.9 \%$ sensitivity, $81.6 \%$ specificity, $38.5 \%$ PPV and $95.9 \%$ NPV for detection of large OV (grade III and IV). On Comparing blood ammonia level and platelet count/splenic diameter ratio as predictors for $\mathrm{OV}$, no significant difference was detected between them.

Table (1): Blood ammonia levels and Platelet count/ splenic diameter ratio of the studied groups.

\begin{tabular}{|l|c|c|c|c|}
\hline & \multicolumn{3}{|c|}{} & \multicolumn{2}{|c|}{ T-test and chi-square } \\
\cline { 2 - 5 } & $\begin{array}{c}\text { Group I } \\
\text { Cirrhotics without } \\
\text { O.V. } \\
(\mathbf{N = 3 0})\end{array}$ & $\begin{array}{c}\text { Group II } \\
\text { Cirrhotics with } \\
\text { O.V. } \\
(\mathbf{N = 7 0})\end{array}$ & Test value & P-value \\
\hline Ammonia $(\mu \mathrm{mol} / \mathrm{l})$ & $5.98 \pm 39.17$ & $9.76 \pm 54.84$ & -8.153 & 0.000 \\
\hline $\begin{array}{l}\text { Platelet count/splenic } \\
\text { diameter Ratio }\end{array}$ & $322.43 \pm 1076.86$ & $251.43 \pm 698.05$ & 6.327 & 0.000 \\
\hline
\end{tabular}

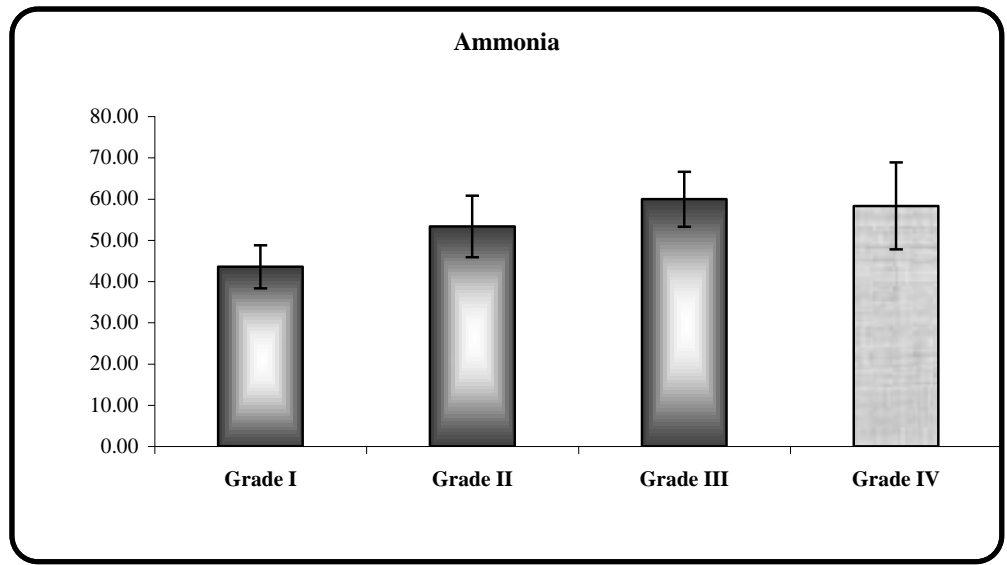

Figure (1): Blood ammonia levels among different grades of oesophageal varices 


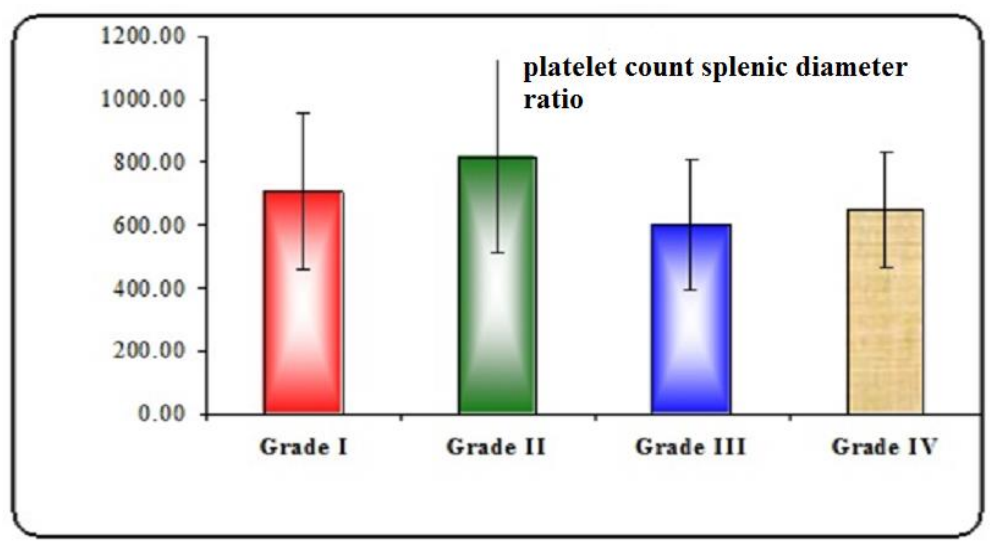

Figure (2): platelet count/splenic diameter ratio among different grades of oesophageal varices

Table (2): Multivariate logistic regression analysis for predictors of presence of oesophageal varices.

\begin{tabular}{|c|c|c|c|c|c|c|c|}
\hline & \multirow{2}{*}{ B } & \multirow{2}{*}{ S.E. } & \multirow{2}{*}{ Wald } & \multirow{2}{*}{ P-value } & \multirow{2}{*}{ Odd } & \multicolumn{2}{|c|}{ 95\% C.I. for Odd } \\
\hline & & & & & & Lower & Upper \\
\hline$\overline{\text { Age }}$ & -0.052 & 0.072 & 0.527 & 0.468 & 0.949 & 0.825 & 1.092 \\
\hline Gender & 0.551 & 1.191 & 0.214 & 0.644 & 1.734 & 0.168 & 17.884 \\
\hline INR & 0.147 & 0.700 & 0.044 & 0.834 & 1.158 & 0.294 & 4.566 \\
\hline Prothrombin time & 0.583 & 0.339 & 2.962 & 0.085 & 1.791 & 0.922 & 3.478 \\
\hline $\mathrm{HB} \%$ & 0.726 & 0.396 & 3.360 & 0.067 & 2.067 & 0.951 & 4.491 \\
\hline PLT & -0.092 & 0.117 & 0.615 & 0.433 & 0.913 & 0.726 & 1.147 \\
\hline Splenic diameter & 1.274 & 1.073 & 1.408 & 0.235 & 3.574 & 0.436 & 29.298 \\
\hline P.V. diameter & 0.632 & 0.608 & 1.078 & 0.299 & 1.881 & 0.571 & 6.199 \\
\hline Child class & -0.278 & 0.956 & 0.084 & 0.771 & 0.757 & 0.116 & 4.929 \\
\hline PHG & 0.352 & 1.446 & 0.059 & 0.808 & 1.422 & 0.084 & 24.216 \\
\hline Blood ammonia & 0.520 & 0.190 & 7.491 & 0.006 & 1.681 & 1.159 & 2.439 \\
\hline platelet count/ splenic diameter ratio & 0.013 & 0.016 & 0.681 & 0.409 & 1.013 & 0.982 & 1.045 \\
\hline
\end{tabular}

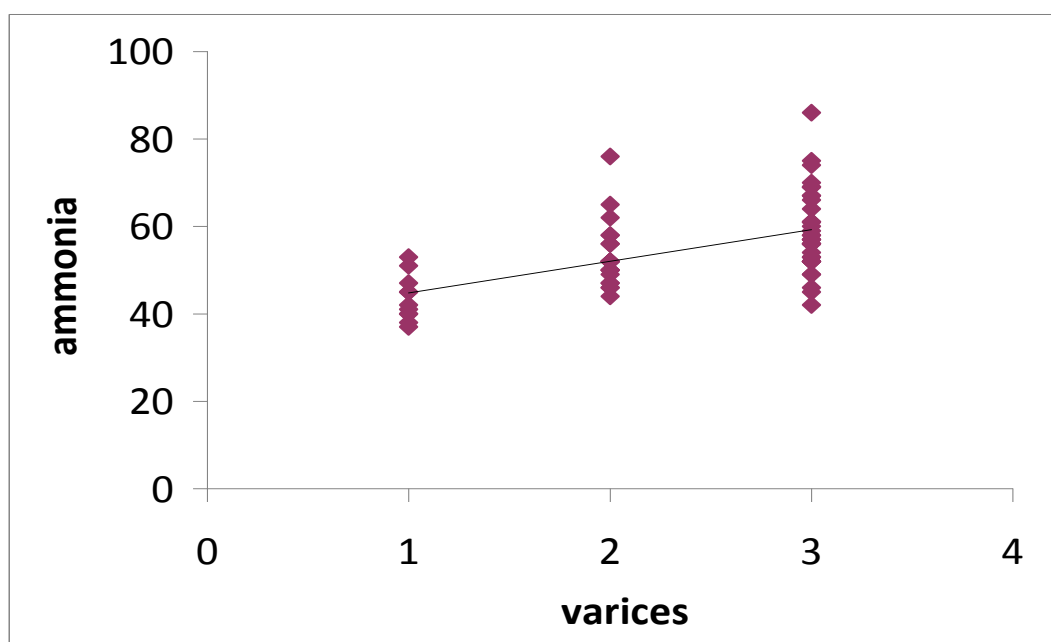

Figure (3): Correlation between blood ammonia levels and size of varices 
Table (3) : Diagnostic performance of platelet count / splenic diameter ratio and blood ammonia level for diagnosis of oesophageal varices.

\begin{tabular}{|l|c|c|c|c|c|}
\hline \multicolumn{1}{|c|}{ Cutoff } & Sens. & Spec. & PPV & NPV & Accuracy \\
\hline platelet count/ splenic diameter ratio $=1073$ & 94.3 & 56.7 & 83.5 & 81.0 & 82.6 \\
\hline Blood ammonia level =48 & 75.7 & 100.0 & 100.0 & 63.8 & 93.2 \\
\hline
\end{tabular}

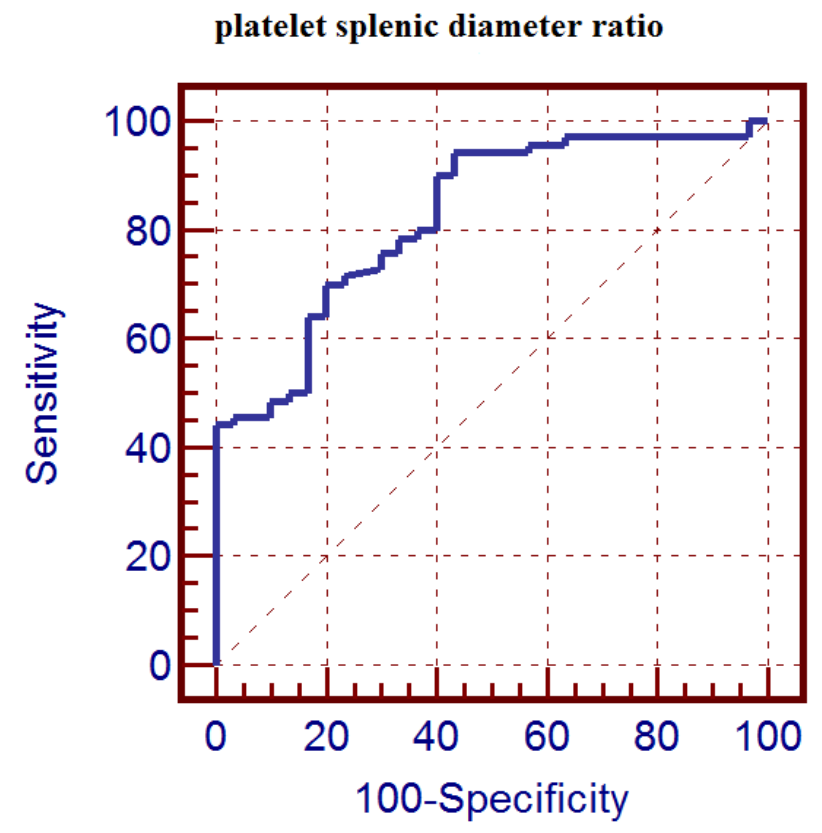

Figure (4): ROC curve of platelet count / splenic diameter ratio for diagnosis of eosophageal varices

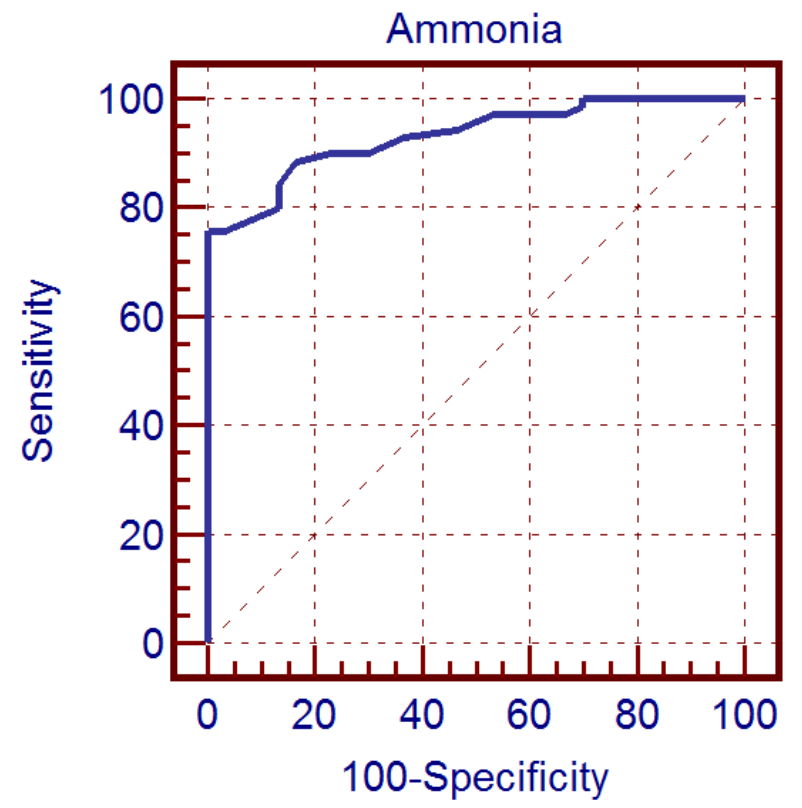

Figure (5): ROC curve of blood ammonia level for diagnosis of eosophageal varices 
Table (4): Diagnostic performance of platelet count / splenic diameter ratio and blood ammonia level for diagnosis of large oesophageal varices (grade III and IV).

\begin{tabular}{|l|c|c|c|c|c|}
\hline \multicolumn{1}{|c|}{ Cutoff } & Sens. & Spec. & PPV & NPV & Accuracy \\
\hline platelet count/splenic diameter ratio = 570 & 76.9 & 81.6 & 38.5 & 95.9 & 84.7 \\
\hline Blood ammonia level =67 & 46.2 & 97.7 & 75.0 & 92.4 & 75.7 \\
\hline
\end{tabular}

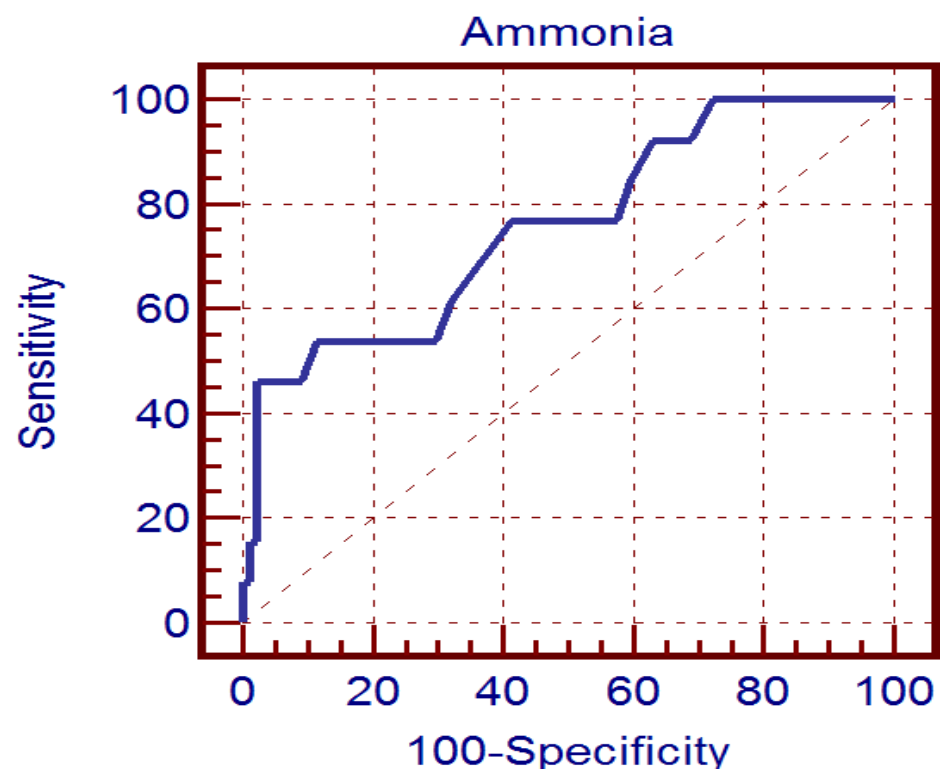

Figure (5): ROC curve of blood ammonia level for diagnosis of large oesophageal varices

Table (5): Comparison between blood ammonia level and platelet count/ splenic diameter ratio as predictors for varices

\begin{tabular}{|c|c|c|c|}
\hline \multicolumn{4}{|c|}{ Ammonia \&platelet count splenic diameter ratio } \\
\hline $\begin{array}{c}\text { Difference } \\
\text { between areas }\end{array}$ & Standard error & $\mathbf{9 5 \%}$ Confidence interval & Significance level \\
\hline 0.092 & 0.085 & -0.075 to 0.258 & 0.280 \\
\hline
\end{tabular}

\section{DISCUSSION}

Oesophageal varices $(\mathrm{OV})$ are the most important and critical portosystemic shunts that develop secondary to portal hypertension. Endoscopic prophylactic band ligation and nonselective beta blockers can minimize the risk of oesophageal bleeding by 50\% [10]. Endoscopic screening of all cirrhotic patients would lead to a large number of unnecessary endoscopies and additional burden to endoscopic units [11]. The development of non-invasive methods for OV prediction could reduce the use of upper gastrointestinal endoscopy in variceal screening and also provide an alternative way to confirm the results of conventional endoscopic diagnosis [12]. A total of 100 adult cirrhotic patients were selected from those attending the department of Hepatology, Gastroenterology and Infectious diseases, Benha University Hospital during the period from January to June 2015. Regarding BAL, the current study showed significant difference between cirrhotic group with $\mathrm{OV}$ and that without $\mathrm{OV}(\mathrm{p}=0.000)$. The mean ammonia level in cirrhotics with OV was $54.84 \mu \mathrm{mol} / \mathrm{l}$ while it was $39.17 \mu \mathrm{mol} / \mathrm{l}$ in those without OV. In addition, BAL was also significantly high in patients with large sized varices (grade III and IV). These results came in agreement with the studies done by Tarantino et al. [7] and 
Khondaker et al. [13] who reported that not only BAL increased in cirrhotic patients with OV but also with those who had large sized varices. BAL at cutoff value $=48 \mathrm{umol} / \mathrm{L}$ had sensitivity $75.7 \%$ and specificity $100 \%$ in detection of OV and at level $=67 \mathrm{umol} / \mathrm{L}$, it had $46.2 \%$ sensitivity, $97.7 \%$ specificity in detecting large sized OV (grade III and IV) in cirrhotic patients of the current work. A study done by Tarantino et al. [7] reported that BAL at cutoff value $=42$ umol/ L had sensitivity of $97 \%$ and specificity of $43 \%$ for detection of OV and Khondaker et al. [13] found that blood ammonia levels at $\geq 63$ umol/l had sensitivity of $95 \%$ and specificity of $50 \%$ in detecting large OV in patients with cirrhosis suggesting its usefulness in identifying patients with large varices who need endoscopies. Also El-Hefny et al. [14] concluded that BAL at cutoff value $77.5 \mathrm{umol} / \mathrm{L}$ had sensitivity $100 \%$ and specificity $95 \%$ for detection of O.V. On the same hand, a study done by Montasser et al. [15] concluded that BAL at cutoff value $133 \mathrm{umol} / \mathrm{L}$ had sensitivity of $100 \%$ and specificity of $96 \%$ in detecting large varices. On contrary to this study, Ramzy et al. [16] concluded that ammonia alone can not predict the presence nor the grade of OV based on the fact, the BAL determination suffers from some limits in its measurements as the collection, handling, storage, and analysis of blood samples are all potential sources of error.

The present study detected significant decrease in the mean values of platelet count/ splenic diameter ratio in cirrhotic patients with varices in comparison to those without varices and moreover in cirrhotics with large varices (grade III and IV) compared to those with small varices (grade I and II). Also, our study found that platelet count/ splenic diameter ratio at cutoff value 1073 had sensitivity $94.3 \%$, specificity $56.7 \%$, PPV $83.5 \%$ and NPV $81 \%$ for detection of $\mathrm{OV}$ and at cutoff 570 had sensitivity $76.9 \%$,specificity $81.6 \%$, PPV $38.5 \%$ and NPV $95.9 \%$ for detection of large varices. This came in concordance with Abo-Alsoud et al. [17] who reported; significant decrease in the mean values of platelet count/ spleen diameter ratio in cirrhotic patients with varices in comparison to other patients without varices, and that the best cutoff value for detection of O.V was 638.9 with sensitivity $100 \%$, specificity $97.5 \%$, PPV $95.2 \%$ and NPV $100 \%$. In contrast to our results, Qamar et al. [18] and Hassan,et al. [19] found that platelets/splenic longitudinal diameter
(PLT/SLD) ratio did not show significant difference in patients with and without OV suggesting that these markers cannot predict the presence of varices. This conflict may be due to difference in severity of liver disease in our patients as $74.29 \%$ of group 2 cases in our study were Child B \&C and $66 \%$ of group 1 were Child A while the previous study included patients of Child "A" and early "B" liver cirrhosis who had less impairment of platelet count which may explain this disagreement. In the current study and according to the multivariate logistic regression analysis for predictors of $\mathrm{OV}, \mathrm{BAL}$ was the only predictor of OV presence. This result was in agreement with Hassan,et al. [19], who reported that BAL, PVD, SVD and SLD were good non-invasive predictors for the presence of $\mathrm{OV}$ in cirrhotics with the superiority of ammonia and PVD, and was in disagreement with Ramzy et al. [16]. Significant positive correlation was noted in our study between BAL and size of varices $(r=.557$ and $P$ value $<0.001$ ) according to Spearman correlation analysis. This finding was comparable with that reported by Tarantino et al. [7] where $\mathrm{r}=0.43$ and $\mathrm{P}$ value was $<0.001$. On the same hand Hassan,et al. [18] reprted that among non-invasive parameters including BAL, PVD, SVD and SLD, only BAL positively correlated with the size of OV and Khondaker et al. [13] observed moderate but significant correlation between BAL and size of OV. when comparing the performance of blood ammonia with the PLT/SLD ratio in the current work, no significant difference was detected ( $p$ value $=$ 0.280 ). A result that came in agreement with Tarantino et al. [7] who do not found dissimilar reliability between BAL and PLT/SLD in prediction of OV. Although the Plts/SLD ratio do not suffer from external confounding factors but its disadvantages are consistent with changes in platalet count either in the form of thrombocytemia that is sometime related to the auto-antibodies presence which turns out in falsely low count of PLTs. or falsely high count of PLTs, as in patients suffering from liver cirrhosis with hepatocelular carcinoma [20].

\section{CONCLUSION}

Blood ammonia level could be a non invasive predictor for the presence of $\mathrm{OV}$ and could be clinically useful, as it correlated with the size of OV. 
Funding: None.

Conflicts of interest: None.

Ethical approval:Approved .

\section{REFERENCES}

1- Dancygier H , Rogart J. Approach to the Patient with Portal Hypertension. In: Clinical Hepatology (v1) 2010; ch.53:p593-602.

2- Bruce R Bacon. Cirrhosis \& its complications. In: HARRISON'S Gastroenterology and Hepatology, Dan Longo, Anthony S. Fauci (2nd edition) Mc Graw Hill: 2010; ch41:p419-433

3- De franchis R. Revising consensus in portal hypertension : Report of the Baveno V consensus workshop on defenitions methodology and therapy in portal hypertension. J Hepatol 2010; 53:762-68.

4- Garcia-Pegan , Garcia-sancho. Functional aspects on pathophysiology in portal hypertension in liver cirrhosis. J Hepatol 2012; 57(2),458-61.

5- Merli M, Nicolini G, Angeloni S, Rinaldi V, De Santis A, Merkel C., et al. Incidence and natural history of small esophageal varices in cirrhotic patients. J Hepatol 2003; 38:266-72.

6- Berzigotti A1, Gilabert R, Abraldes JG, Nicolau C, Bru C, Bosch J., et al. Non invasive prediction of clinically significant portal hypertension and oesophageal varices in patients with compensated liver cirrhosis. Am.J. Gastroenterol 2008; 103(5),1159-67.

7-Tarantino G1, Citro V, Esposito P, Giaquinto S, de Leone A, Milan G., et al. Blood ammonia levels in liver cirrhosis: A clue for the presence of portosystemic collateral veins. $B M C$ Gastroenterol 2009;9:21.

8- De Franchis R. Evolving consensus in portal hypertension; report of the Baveno IV consensus workshop on methodology of diagnosis, therapy in portal hypertension and grading of esophageal varices. J Hepatol 2005; 43: 167-76.

9- Vananken HC , Scphorst ME, A kinetic determination of ammonia in plasma. Clin Chem Acta 1974; 56: 151-157.

10- Giannelli V, Lattanzi B, Thalheimer U, Merli, M. Beta-blockers in liver cirrhosis. Ann. Gastroenterol 2014; 27 (1): 20-26.

11- De Franchis R. Noninvasive diagnosis of esophageal varices: is it feasible? Am. J. Gastroenterol 2006; 101: 25202.
12- Wang L, Hu J, Dong S, Jian YC, Hu L, Yang G.,et al. Noninvasive prediction of large esophageal varices in liver cirrhosis patients. Clin Invest Med 2014; 37 (1): E38-46.

13- Khondaker M, Ahmad N, Al-Mahtab M, Sumi SA. Correlation between blood Ammonia Level and oesophageal Varices. Euroasian J HepatoGastroenterol 2013; 3: 10-14.

14- El-Hefny N, Hareedy M and Abdel-Aal R. Blood ammonia level and other non invasive parameters as indicators of portosystemic collaterals in cirrhotic patients: Thesis submitted for partial fulfillment of MSC degree in internal medicine. Assuit university 2013.

15- Montasser M, Abdella H, Samy A. Evaluation of venous ammonia level, splenic longitudinal diameter and portal vein diameter as non invasive indicator for portosystemic collaterals in Egyptian cirrhotic patients. Open journal of Gastroenterology 2014; (4) 265-74.

16- Ramzy I, Hafez HA, Madani H, Sanad N. Predictive Value of Non-Invasive Blood Ammonia Level for the Presence of Oesophageal Varices in Egyptian Patients With Liver Cirrhosis. Journal of Gastroenterology and Hepatology Research 2015; 4(5): 1613-1617 Available from: URL: http://www.ghrnet.org/index.php/joghr/article/vie w/1207.

17- Abo-Alsoud A.A; Badawy A.M; Sonbol A.A; Ayad M.E. Study of the Relationship between Blood Ammonia Level and Esophageal Varices in Patients with Liver Cirrhosis Afro-Egypt $J$ Infect Endem Dis 2015; 5(2): 78-85.

18- Qamar AA, Grace ND, Groszmann RJ, GarciaTsao G, Bosch J, Burroughs AK., et al. Platelet count is not a predictor of the presence or development of Gastroesophageal varices in cirrhosis. Hepatology 2000; 47: 153-159.

19- Hassan EA; Abd El-rehim AS ; Sayed ZA; Kholef EF; Hareedy MA ; Abd EL-aaL RF. NonInvasive Parameters of Oesophageal Varices Diagnosis: Which Sensitive and Applicable; A Pilot Study. J Liver 2015; 4:3,http://dx.doi.org/10.4172/21670889.1000182 .

20- Giannini EG, Zaman A, Kreil A, Floreani A, Dulbecco P, Testa E., et al. Platelet count/spleen diameter ratio for the noninvasive diagnosis of esophageal varices: results of a multicenter, prospective, validation study. Am J Gastroenterol 2006;101:2511-2519. doi: 10.1111/j.15720241.2006.00401_6.x. [PubMed] [Cross Ref] 